\title{
NOTICIARIO
}

\section{Instrumentos para siega y procesado de plantas desde el Calcolítico al Bronce antiguo de Chibanes (Palmela, Portugal)}

\author{
Harvesting and plant processes tools from the Chalcolithic to the Ancien Bronze Age of \\ Chibanes (Palmela, Portugal)
}

Ignacio Clemente-Conte (*)

Niccolò Mazzucco (*)

Joaquina Soares (**)

\section{RESUMEN}

El análisis funcional ha reconocido rastros de uso en artefactos de sílex (foicinhas) que caracterizan la identidad socio-cultural del III milenio cal BC en la Estremadura portuguesa. Esos artefactos tienen rasgos tecnológicos y tipológicos específicos y su funcionalidad ha sido discutida durante mucho tiempo sin resultados positivos. El presente estudio funcional permitió su clasificación como elementos de hoz, proporcionando información sobre las prácticas agrícolas durante el Calcolítico en Portugal y nuevos enfoques sobre la biografía de las foicinhas. Este trabajo supone un ejemplo más de la aportación del análisis funcional al conocimiento de los aspectos socio-económicos en las sociedades del pasado.

\begin{abstract}
Micro-wear analysis has been applied to one of the characteristic flint tools types (foicinhas) of the III millennium cal BC in Portuguese Estremadura. Foicinhas have a specific technological and typological character and their function has been discussed long time ago
\end{abstract}

(*) Departamento de Arqueología y Antropología, Institución Milà y Fontanals, Consejo Superior de Investigaciones Científicas (IMF-CSIC). C/ Egipciaques 15. 08001 Barcelona. España.

Correos e.: Ignacio@imf.csic.es; niccomazzucco@imf.csic.es

(**) Museu de Arqueologia e Etnografia do Distrito de Setúbal. Av. Luisa Todi 162. 2900-451 Setúbal. Portugal. Centro de Arqueologia da Faculdade de Letras da Universidade de Lisboa.

Correos e.: cea.maeds@mail.telepac.pt; joaquinasoares1@gmail.com Recibido: 22-V-2013; aceptado: 6-IX-2013. without any positive results. The present use-wear study makes clear that they are sickles. This provides information about the agricultural practices of the Portuguese Chalcolithic and gives new insights as to the biography of the foicinhas. This paper highlights the contribution of micro-wear analysis for an integrated socioeconomic approach to chipped stone tools.

Palabras clave: Análisis funcional; Elementos de hoz; Calcolítico; Península Ibérica; Tratamiento térmico, Tri1lo.

Key words: Use-Wear analysis; Sickle implements; Chalcolithic; Iberian Peninsula; Heat treatment; Tribulum.

\section{INTRODUCCIÓN: ANTECEDENTES}

Afonso do Paço (Paço et al. 1959) clasificó como foicinhas una serie de piezas de sílex con formas ovales y retoque plano, invasor y cubriente, presentes en gran parte de los yacimientos del III milenio BC de la Estremadura portuguesa. Eduardo da Cunha Serrão (Serrão 1978) criticó esta atribución funcional y propuso denominarlas lâminas de faca (hojas de navaja) con filo convexo, en base a una analogía etnográfica con los Ipiutak, esquimales de Alaska, de inicios del I milenio DC (Larsen y Rainey 1948) y sin comparar los respectivos contextos socioeconómicos. Este mismo autor, sin preparación, ni experiencia previa para analizar rastros de uso, observó con lupa binocular artefactos procedentes de los sitios 
de Olelas y Negrais concluyendo que no presentaban huellas relacionadas con la siega de cereales (Serrão 1978: 18).

Más de treinta años del inicio de esta 'ingenua' polémica ha sido posible destacar la importante función económica desempeñada por este tipo de instrumento, un buen indicador de la identidad sociocultural de la formación social calcolítica de la Estremadura portuguesa. Los elementos de hoces ovoides, o foicinhas, así como la identificación de elementos de trillo están indicando la importancia de las prácticas agrícolas en una economía agropecuaria productora de excedentes.

Estas foicinhas, incluidas en el grupo de piezas foliáceas, podrían representar el útil más característico del Calcolítico de la Estremadura portuguesa (Carvalho 1996; Amaro 2004/2005). Por lo general están manufacturadas a partir de lascas grandes o láminas cortas de sílex, talladas por percusión directa y retocadas bifacialmente por presión tras un calentamiento del soporte. El macro grupo de los foliáceos suele dividirse en: foicinhas, puntas de seta y otros tipos que incluyen alabardas y puñales. Estas últimas son piezas con una esmerada elaboración y poco frecuentes.

Podemos observar la evolución diacrónica del grupo tecno-tipológico de los foliáceos en la secuencia estratigráfica del sitio de Leceia (Cardoso et al. 1983/84, 1996) desde la segunda mitad del IV milenio hasta mediados o el tercer cuarto del III milenio cal BC. La primera constatación es la estabilidad de la estructura tecno-tipológica de la industria lítica tallada durante las tres fases principales de la dinámica ocupacional del sitio: Neolítico final (2. ${ }^{\mathrm{a}}$ mitad del IV milenio BC), Calcolítico antiguo (1. ${ }^{\mathrm{a}}$ mitad del III milenio cal BC) y Calcolítico pleno (mediados/3 ${ }^{\text {er }}$ cuarto del III milenio cal BC). El grupo de foliáceos aparece al final del Neolítico con el 7,9\% de las piezas retocadas: 5,8\% corresponden al subgrupo de foicinhas y $2,1 \%$ al de puntas de seta. En la 1. ${ }^{\mathrm{a}} \mathrm{mitad}$ del III milenio, en el Calcolítico antiguo, se registra su máximo desarrollo con el 25,3\% de los retocados, siendo un $20,3 \%$ foicinhas y un $5 \%$ puntas de seta. Al parecer los instrumentos dedicados a la agricultura cuadruplican los dedicados a actividades cinegéticas o guerreras. A partir de mediados del III milenio las piezas foliáceas se reducen a un $15,8 \%$, de las cuales un $10,3 \%$ son hoces. En el último cuarto del III milenio, en el Bronce Antiguo, las foicinhas entran en desuso y cambiando la morfología y tecnología de los elementos de hoz documentados.

El estudio de la industria lítica de la ocupación del sitio del Pedrão (Setúbal, Portugal), datada a inicios del III milenio (Calcolítico antiguo), mostró una fuerte presencia del grupo de foliáceos. Eran el $36,4 \%$ de la totalidad de los artefactos retocados: $31 \%$ pertenecen al subgrupo de puntas de seta; $4,7 \%$ al de foicinhas y $0,7 \%$ al de alabardas (Soares y Tavares da Silva 1975).

Los resultados de los análisis de las industrias líticas de los sitios de Leceia (Península de Lisboa) y de Pedrão (Península de Setúbal) establecen una relación porcentual entre puntas de seta y foicinhas, generalizada en Estremadura desde finales del IV y durante el III milenio cal BC. Esa relación permite acercarnos a los aspectos económicos del contexto analizado. La principal innovación que se presenta en este trabajo es la determinación funcional de las hoces y de sus cambios en forma y manufactura desde el Calcolítico antiguo hasta el Bronce Antiguo en el sitio de Chibanes.

\section{EL POBLADO FORTIFICADO DE CHIBANES}

El poblado fortificado de Chibanes (Fig. 1) se localiza en la Pre-Arrábida (Portugal), rodeado de suelos de elevada calidad agrícola, arcillosos y espesos, resultantes de la descomposición de calcarenitos miocenos. Para su explotación debió de usarse el arado (Soares 2003). La vocación agrícola, de silvicultura y pastoril de Chibanes queda también documentada por la información arqueobotánica. Esta procede de muestras de la ocupación de la $1 .^{\mathrm{a}}$ mitad y mediados del III milenio cal BC del poblado, el período de máximo desarrollo de las foicinhas (Tereso 2014: 173-180), así como de los diagramas polínicos (zona polínica CAR C) obtenidos en las turberas de las lagunas de Estacada e Apostiça en la Península de Setúbal (Queiroz 1999). Ambas series de datos permiten reconstruir un territorio de agricultura intensiva instalada a partir de extensas deforestaciones en los alrededores de Chibanes. Existirían también manchas de sotobosque de tipo esclerófilo mediterráneo donde el madroño (Arbutus unedo) parece haber sido favorecido antrópicamente dado su predominio en las muestras de antracología analizadas. La presencia de habas (Vicia faba) en 

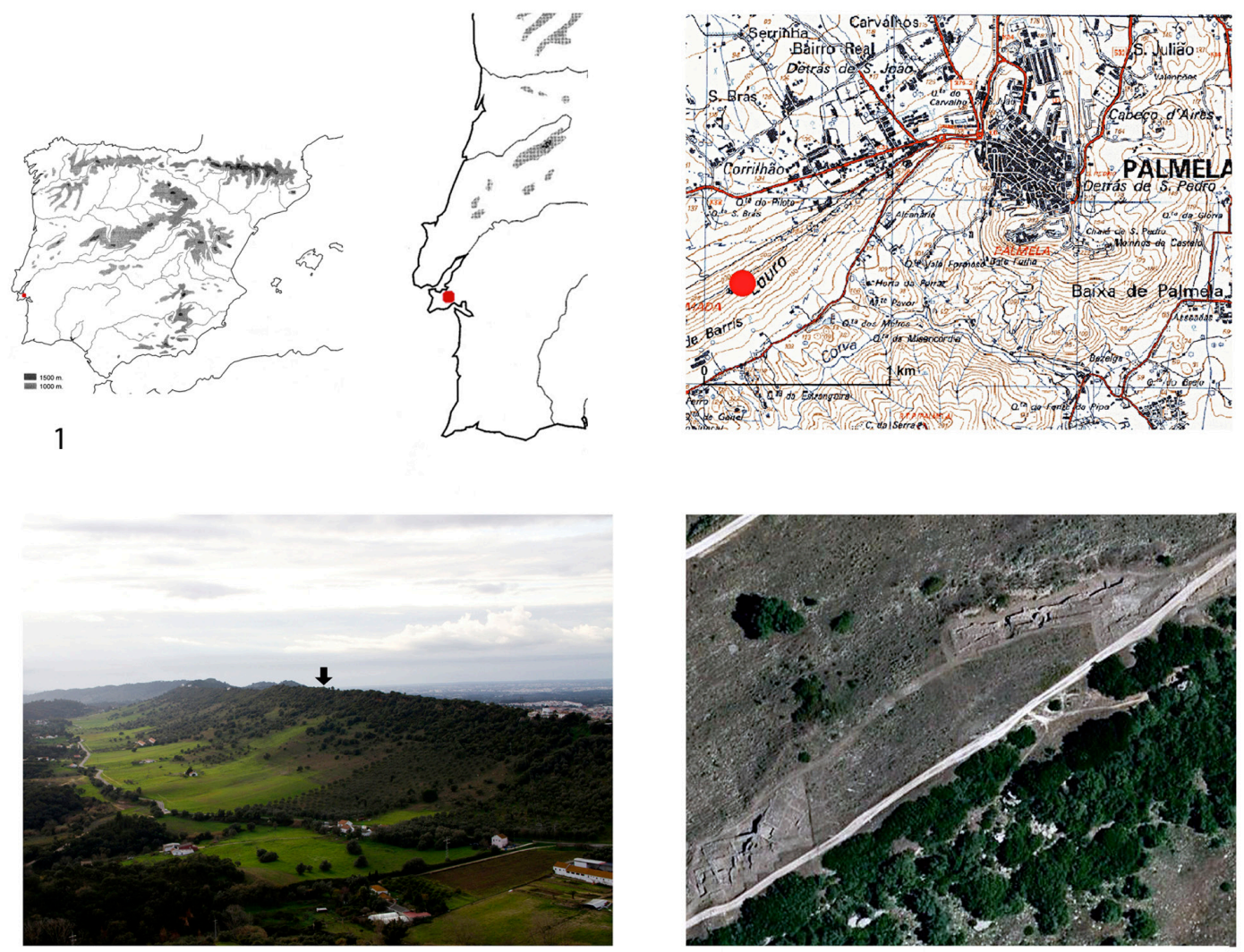

2

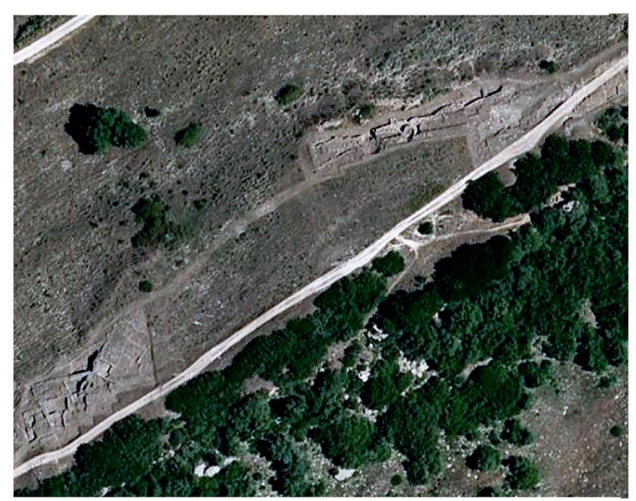

3

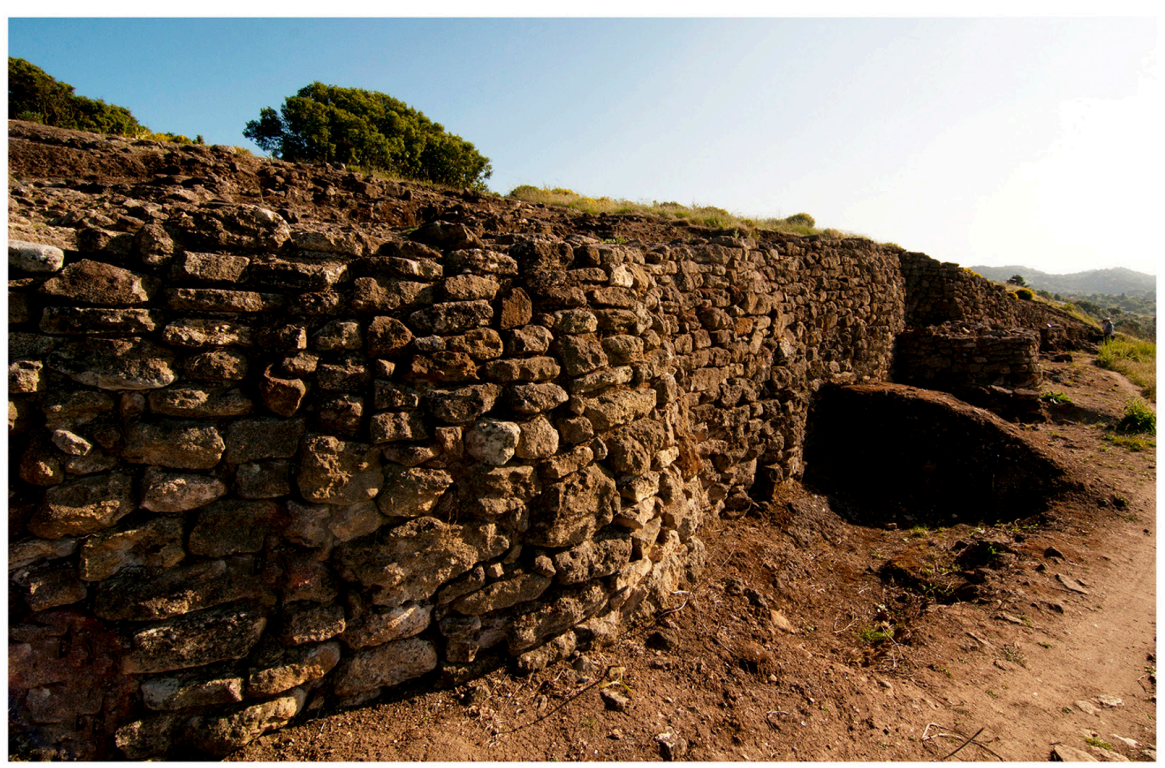

4

Fig. 1. 1. Localización del poblado fortificado de Chibanes en la Península Ibérica y en la Serra do Louro (término municipal de Palmela, Portugal) (el mapa proviene de la Carta Militar de Portugal Série M78- Folha 38-I-Setúbal); 2. Serra do Louro desde el SE; 3. Vista aérea del poblado (Google maps); 4. Vista de la muralla del III milenio cal BC desde el NE (fotos 2 y 3 J. Soares).

Trab. prehist., 71, N. ${ }^{\circ}$ 2, julio-diciembre 2014, pp. 330-342, ISSN: 0082-5638 doi: $10.3989 /$ tp.2014.12137 
la fase IA1 puede expresar un cultivo de alternancia en una agricultura supuestamente de base cerealista.

Los factores topográficos influyeron también en la ubicación escogida para el poblado que se asienta en la propia cima de la Sierra del Louro, dominando visualmente los estuarios de los ríos Tajo y Sado. En este lugar se levantaron tres fortificaciones en discontinuidad estratigráfica: calcolítica, de la Edad del Hierro y romano-republicana (Tavares da Silva y Soares 1997, 2012). Nos interesa en este trabajo la fortificación más antigua con ocupaciones del Calcolítico y Bronce antiguo (Tavares da Silva y Soares 2014). Se inicia en el $1^{\text {er }}$ cuarto del III milenio cal BC con la construcción de una fortificación arqueada, reforzada por baluartes, que se extendió por 1 ha (fase IA1). El poblado se cierra por el Norte mediante una muralla reforzada por bastiones; en su límite meridional aprovecha un escarpado natural inexpugnable.

Como recordaremos, los elementos de hoz ovales (foicinhas) tienen su mayor desarrollo durante el Calcolítico de Estremadura, cuyo modelo socioeconómico decae a partir de mediados del III milenio. Ese declive se documenta en los primeros derrumbes de la muralla y sus correlativos incendios (fase IA2). Un seísmo regional, también reconocido en otros sitios coetáneos como Leceia y Zambujal, desencadenó la catástrofe. No parece haber influenciado en el abandono del poblado aunque coincide con las primeras señales de agotamiento del modo de producción calcolítico de la Estremadura portuguesa (Soares 2003). Algunos tramos derrumbados de la muralla no fueron reparados.

La fase IB del Calcolítico pleno de mediados del III milenio se caracteriza por la divulgación de la metalurgia de cobre y por una cerámica profusamente decorada con motivos de 'hoja de acacia' y 'crucífera'.

La fase IC, datada en la transición entre el III y último cuarto del III milenio cal BC, corresponde al colapso del modo de producción calcolítico (Soares y Tavares da Silva 2010; Soares 2014) y al florecimiento de la cerámica campaniforme de estilo Palmela (Soares y Tavares da Silva 1984). Los instrumentos en piedra tallada pierden importancia y los de tipo foicinha son residuales en algunos contextos, más conservadores, como parece haber sido el sitio de Chibanes.
Durante la fase ID de Chibanes, a finales del III e inicios del II milenio cal $\mathrm{BC}$, continúa el abandono del poblado. La población se dispersa por las tierras bajas de la Estremadura portuguesa, territorios más extensos que excluyen la expresión del conflicto intergrupal a escala local. En esta zona se desarrolla la cerámica campaniforme de estilo Palmela evolucionado o 'epicampaniforme’ muy rica en decoración incisa. El registro funerario documenta otra estrategia de organización sociopolítica, más jerarquizada, con concentración de poder en manos de 'jefes' guerreros. Se evidencia cómo en el Bronce antiguo surgen nuevas realidades políticas de tipo jefatura que abren paso al estado arcaico. En Chibanes los elementos de hoz tipo foicinhas son sustituidos por denticulados sobre fragmentos de láminas y/o lascas. Estos artefactos aparecen ya en la fase anterior (Grupo Palmela del Horizonte Campaniforme) y prosiguen en el Bronce final en hábitats cuyos pobladores basaron su economía en el cultivo de cereales. En Tapada de Ajuda en Lisboa (Cardoso et al. 1986), se asocian a la siega y al trillado de cereales. También están presentes, aunque en menor densidad en el Alentejo, en el litoral como en Cerradinha (Tavares da Silva y Soares 1978) o en el interior como Casarão da Mesquita 3 (Santos et al. 2008).

\section{MATERIALES Y ANÁLISIS}

El estudio traceológico se basa en una muestra de 14 artefactos de características tecnomorfológicas similares. Estos representan tan solo el $6,5 \%$ del total (215) de los artefactos recuperados durante las excavaciones de los contextos del III milenio cal BC de Chibanes (fases 1A-D). Estos materiales están depositados en el Museu de Arqueologia e Etnografia do Distrito de Setúbal. Las piezas por fases eran insuficientes para extraer conclusiones estadísticamente consistentes. En los contextos de la 1. ${ }^{\mathrm{a}}$ mitad del III milenio (fase IA) y del principio de la segunda (fase IB) hay 64 artefactos, de los cuales 25 son retocados. El grupo tipológico de foliáceos tiene 16 de los que 11 son de tipo foicinha. De la ocupación del final de la 2. ${ }^{a}$ mitad del III milenio cal BC (fases IC y ID) proceden 151 artefactos. Entre los 58 retocados solo hay 9 ejemplares $(15,6 \%$ de los retocados) del grupo tipológico de los foliáceos. 
Un fragmento sin rastros de uso corresponde al tipo foicinha.

Los instrumentos estudiados tienen forma tendente a rectangular $\mathrm{y}$, mayoritariamente, retoque plano y profundo en ambas caras. La formatización de estos instrumentos parte de una lasca grande extraída del núcleo por percusión directa tratada térmicamente antes del retoque. De las fases IA y IA2 (1. ${ }^{\mathrm{a}}$ mitad del III milenio cal BC) proceden diez de los instrumentos estudiados (Fig. 2: 1-10); un par de la fase IC, nivel campaniforme o grupo Palmela (2. ${ }^{\mathrm{a}}$ mitad del III milenio cal BC) (Fig. 2: 11-12) y otro par de la fase ID (final del III inicios del II milenio cal BC) o Bronce antiguo (Fig. 3).

El tratamiento térmico se documenta en los negativos de los retoques bifaciales, planos y profundos que se disponen por regla general en ambos filos de los artefactos. Este retoque se realiza presionando desde el vértice del filo hacia el in- terior de la pieza con un retocador hasta ahora indeterminado. El tratamiento térmico reduce la fuerza que hace falta aplicar para conseguir retoques de esa profundidad y la pérdida de materia en cada reavivado del filo. El tratamiento térmico se refleja en el brillo intenso y graso que ocupa toda la superficie de esos negativos (Figs. 2-4-6). En cambio otras alteraciones térmicas como cambios de coloración, manchones negros de resinas, fracturas, resquebrajaduras y 'cúpulas térmicas' (Clemente-Conte 1995, 1997; Gibaja-Bao y Clemente-Conte 1997) son posteriores al tratamiento térmico, retoque y uso de los instrumentos. El que las 'cúpulas' o levantamientos térmicos corten y fracturen los negativos prueba su carácter fortuito. Se producen al abandonar o depositar las piezas en fuentes de calor $u$ hogares.

Hemos documentado rastros relacionados con el corte de plantas no leñosas a modo de hoz (cuchillo/hoz) en la mayoría de los artefactos
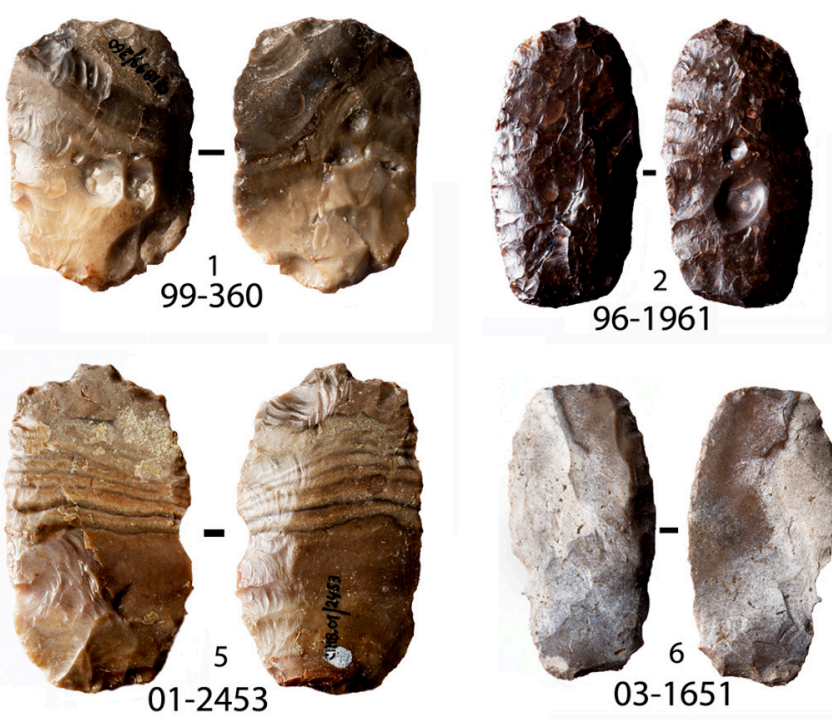

$01-2453$
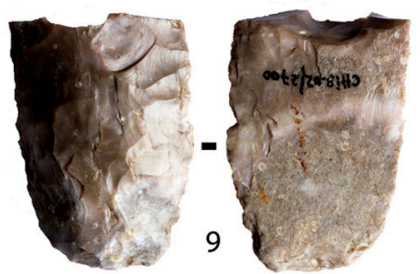

02-2700

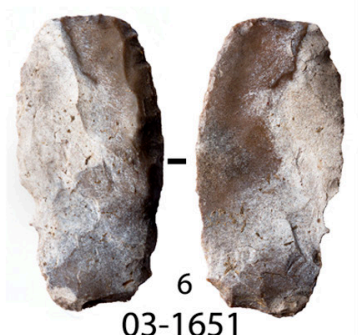

03-1651
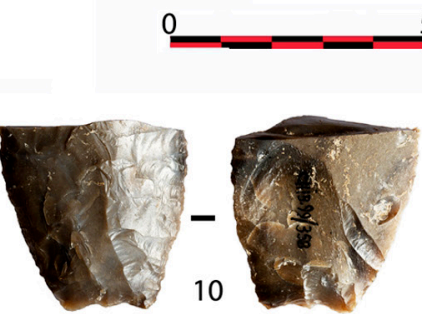

99-358

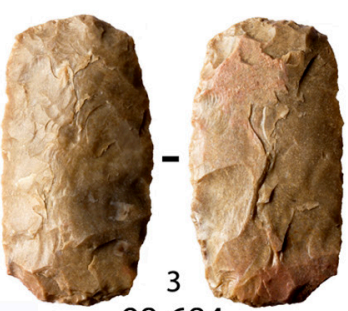

98-684

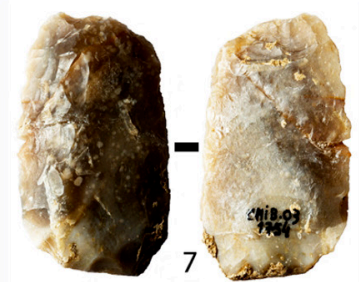

03-1754 $5 \mathrm{~cm}$

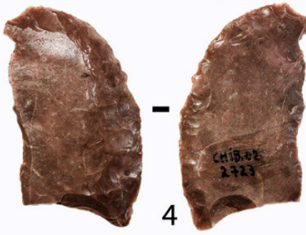

02-2723

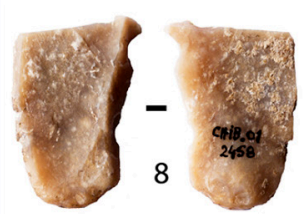

01-2168

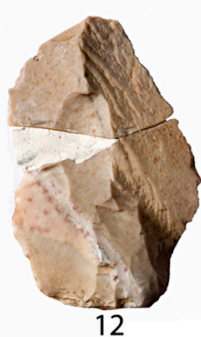

02-2786

Fig. 2. Instrumentos de sílex del poblado fortificado de Chibanes (término municipal de Palmela, Portugal) con rastros atribuibles a siega y corte de cereales o de plantas no leñosas: 1-10 pertenecen a las Fases IA y IA2 - Calcolítico, datadas en la 1. ${ }^{\mathrm{a}}$ mitad del III milenio cal BC; 11 y 12 de la Fase ID - Edad del Bronce antiguo.

Trab. prehist., 71, N. ${ }^{\circ}$ 2, julio-diciembre 2014, pp. 330-342, ISSN: 0082-5638 doi: $10.3989 /$ tp. 2014.12137 

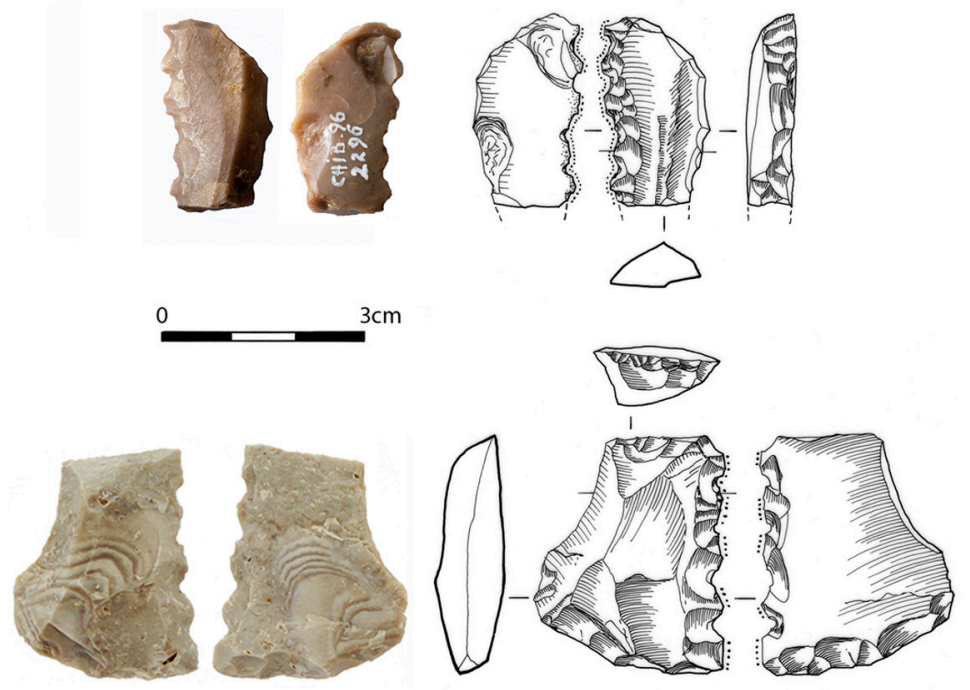

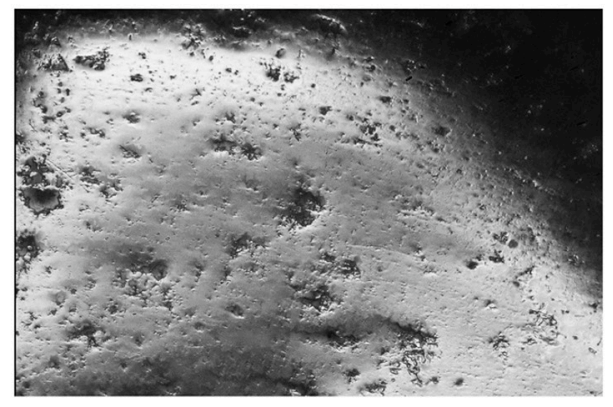

$150 X$

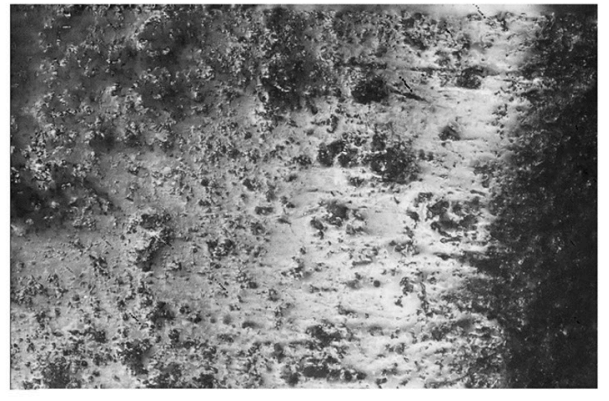

$150 X$

Fig. 3. Dos dientes de hoz denticulados en sílex, recuperados en el poblado fortificado de Chibanes (término municipal de Palmela, Portugal) en la fase correspondiente al Bronce Antiguo. Dibujo de Fernanda de Sousa.

analizados. Sin embargo varían la distribución de rastros y la intensidad de uso. Algunos solo presentan un filo activo (96-1961, 98-684, 01-2453, 02-2723). En un par hay rastros de uso en dos filos (Fig. 2: 99-360 y 03-1651). Llama la atención que en ninguna de las piezas de este tipo fracturadas (99-347, 99-358, 01-2445, 02-2700 y 02-2886) se hayan registrado rastros atribuibles al corte de plantas. Dos carecen de cualquier alteración por uso. Probablemente se fracturaron en el proceso de formatización y se abandonaron. Sin embargo en 01-2445 se observan probables rastros de una acción transversal en el filo derecho y en 02-2700 (Fig. 2) huellas en ambos filos de haberse utilizado en una acción transversal sobre una materia rígida de dureza media, probablemente madera.

El retoque plano y profundo que formatiza los filos se realizó antes de que se enmangaran y utilizaran. Al tratarse de soportes con filos rectos y largos, entre 5 y $6 \mathrm{~cm}$, podrían haber sido enmangados individualmente o de dos en dos en la punta de un mango recto o ligeramente inclinado. En el extremo de algunos filos de estos instrumentos se registran rastros mucho más abrasivos que en el resto. Esto sucede con 03-1651 (Fig. 2:
6) cuyos dos filos utilizados siguen ese parámetro. En 99-360 (Fig. 4), ambos filo presentan rastros típicos del corte de plantas (cereales) pero en uno tienen aspecto más rugoso y el brillo más mate por abrasión con elementos minerales de la tierra (Clemente-Conte y Gibaja-Bao 1998). Les atribuimos al corte de la paja del cereal al ras del suelo (Gibaja-Bao y Clemente-Conte 1996; Gibaja-Bao et al. 1997).

Ya habíamos documentado esos rastros en instrumentos de yacimientos neolíticos para cortar tallos de paja sobre el suelo y/o para utilizarse como trillos (Anderson e Inizan 1994; ClementeConte y Gibaja-Bao 1995, 1998; Clemente-Conte et al. 1999; Gurova 2001; Anderson et al. 2004). Así pues en Chibanes, durante el Calcolítico antiguo, también se intentaron cortar los tallos a ras de suelo con las hoces.

Otro aspecto interesante de las hoces es el reavivado de sus filos con extracciones largas obtenidas por presión. Gracias a ella los filos mantienen el ángulo plano que les hace de nuevo efectivos. Cuando se vuelven a embotar, el reavivado emplea extracciones más cortas y en ángulo más abrupto. La reiteración de esta secuencia hace que el filo sea cada vez menos cortante 


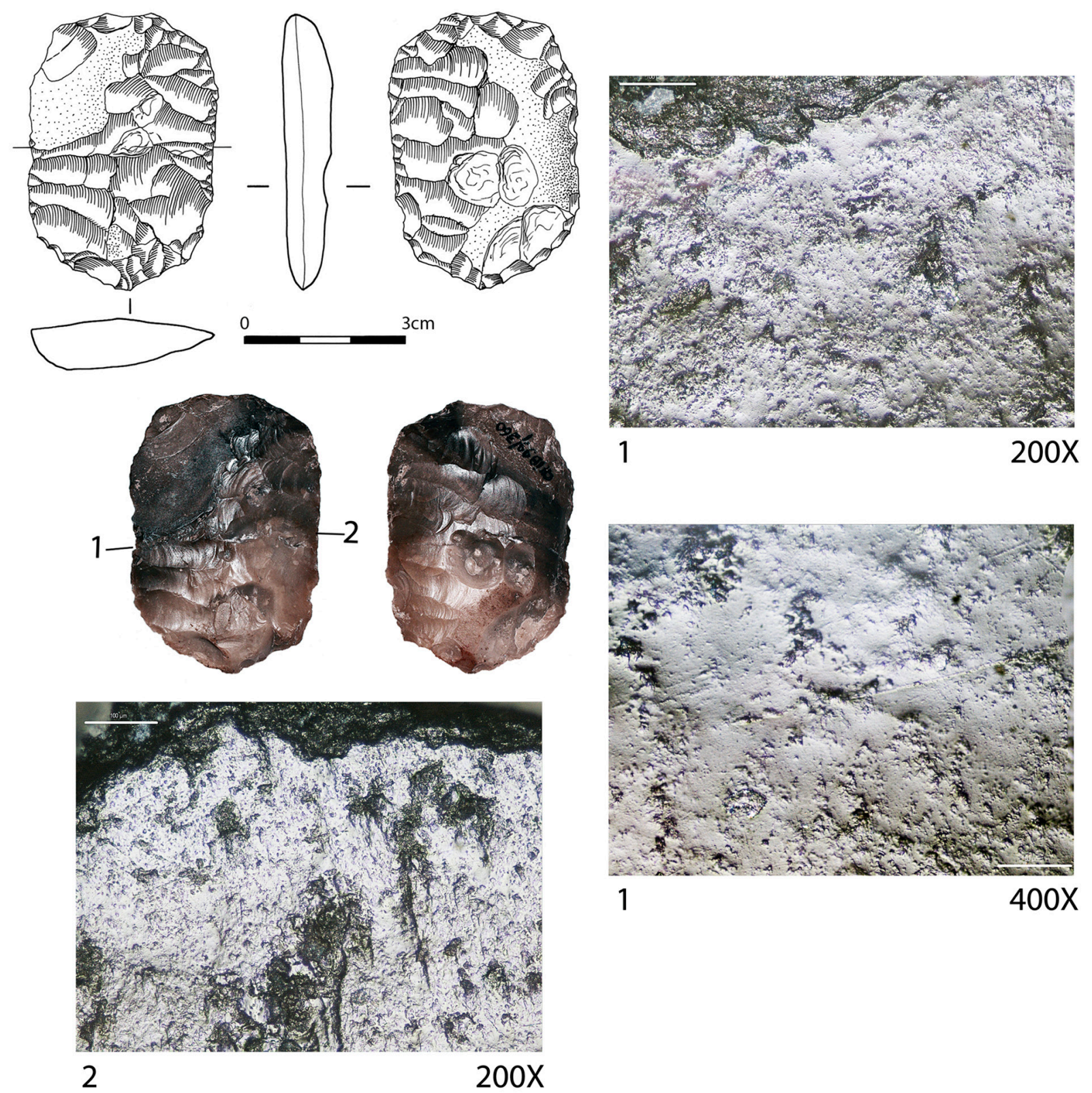

Fig. 4. Foicinha (Chib.99.360), lasca de sílex con retoque plano muy profundo y bifacial, correspondiente al Calcolítico del poblado fortificado de Chibanes (término municipal de Palmela, Portugal). El filo reproducido en las fotos presenta rastros atribuidos al corte de plantas no leñosas (1) y el contrario (2) micropulido más rugoso y mate por contacto con plantas y elementos minerales de la tierra. Se advierten 'cúpulas térmicas'. Dibujo de Fernanda de Sousa.

hasta dejar de utilizarse o girarse para cambiar el lado enmangado. Hemos detectado el reavivado en la mayoría de los filos activos (Fig. 5) y en el izquierdo de 01-2453 en dos momentos distintos. Obsérvese como el filo se escalona mientras, en el derecho, el retoque, en cara ventral, es simple de extracción única (Fig. 6). Tras el reavivado en los negativos de las extracciones se observa con claridad un lustre térmico fresco (Fig. 6: 1). Tras el uso la fricción altera de nuevo la superficie y redondea las aristas (Fig. 6: 2). Un contacto más intenso con los tallos de la planta cubre completamente la superficie de un micropulido brillante, liso y de trama compacta (Fig. 6: 3). Además, en

Trab. prehist., 71, N. ${ }^{\circ}$ 2, julio-diciembre 2014, pp. 330-342, ISSN: 0082-5638 doi: $10.3989 /$ tp.2014.12137 
una zona cercana al filo izquierdo de la misma hoz, hemos documentado unas 'manchas' de pulido en espejo (Fig. 6: 4) o de tipo ' $G$ ' (Moss 1987) relacionables con el contacto y/o fricción con el mango (Rots 2010), entre otros aspectos. Destacamos que el filo derecho de este instrumento ha sido formatizado por retoque antes de
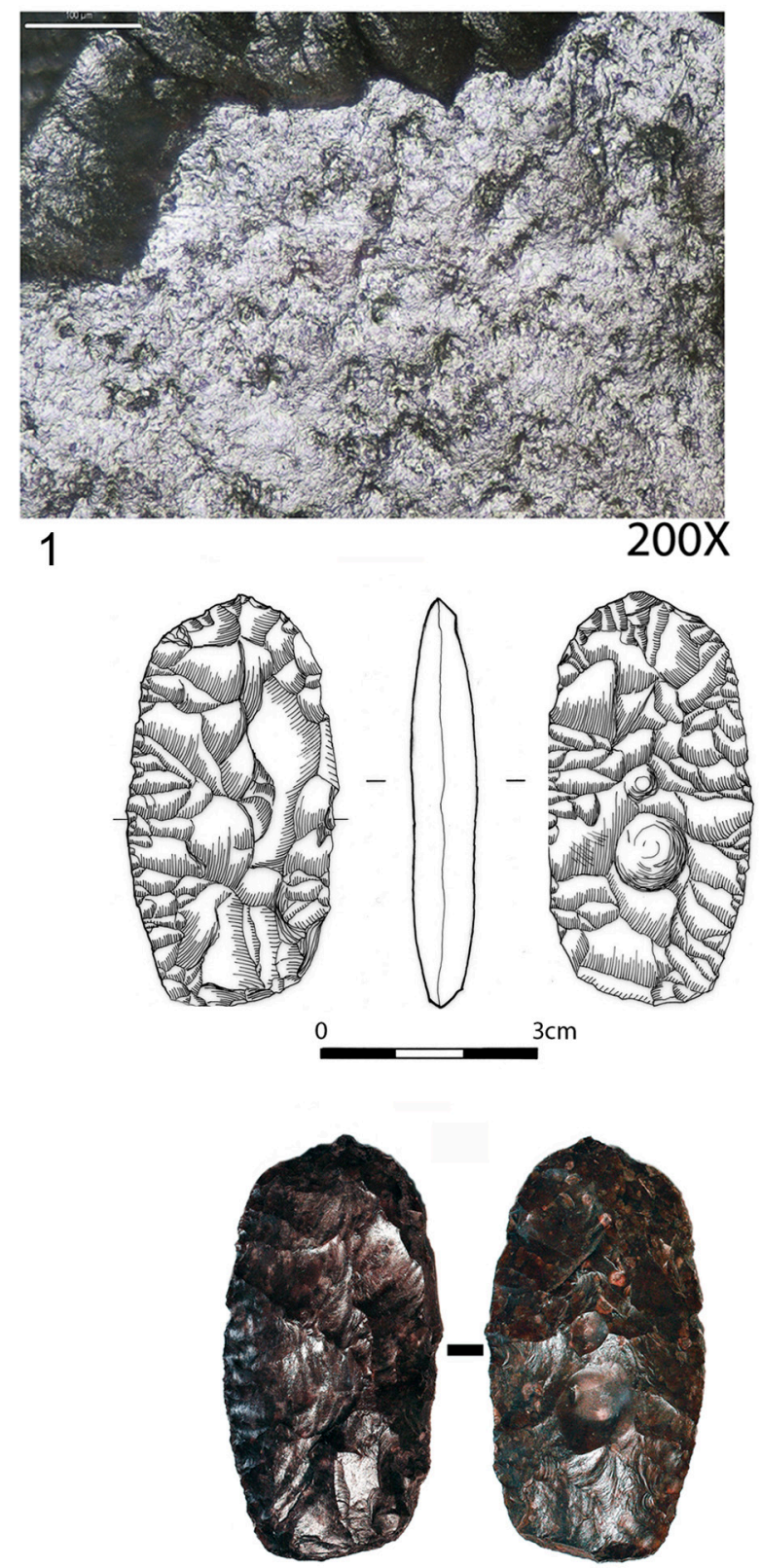

Fig. 5. Foicinha sobre lasca de sílex retocada (Chib.96-1961), correspondiente al Calcolítico del poblado fortificado de Chibanes (término municipal de Palmela, Portugal): 1 lustre térmico en negativo de retoque; 2 y 3 micropulido de corte de plantas no leñosas con distribución en negativos de reavivados. Se advierten "cúpulas térmicas". Dibujo de Fernanda de Sousa. 

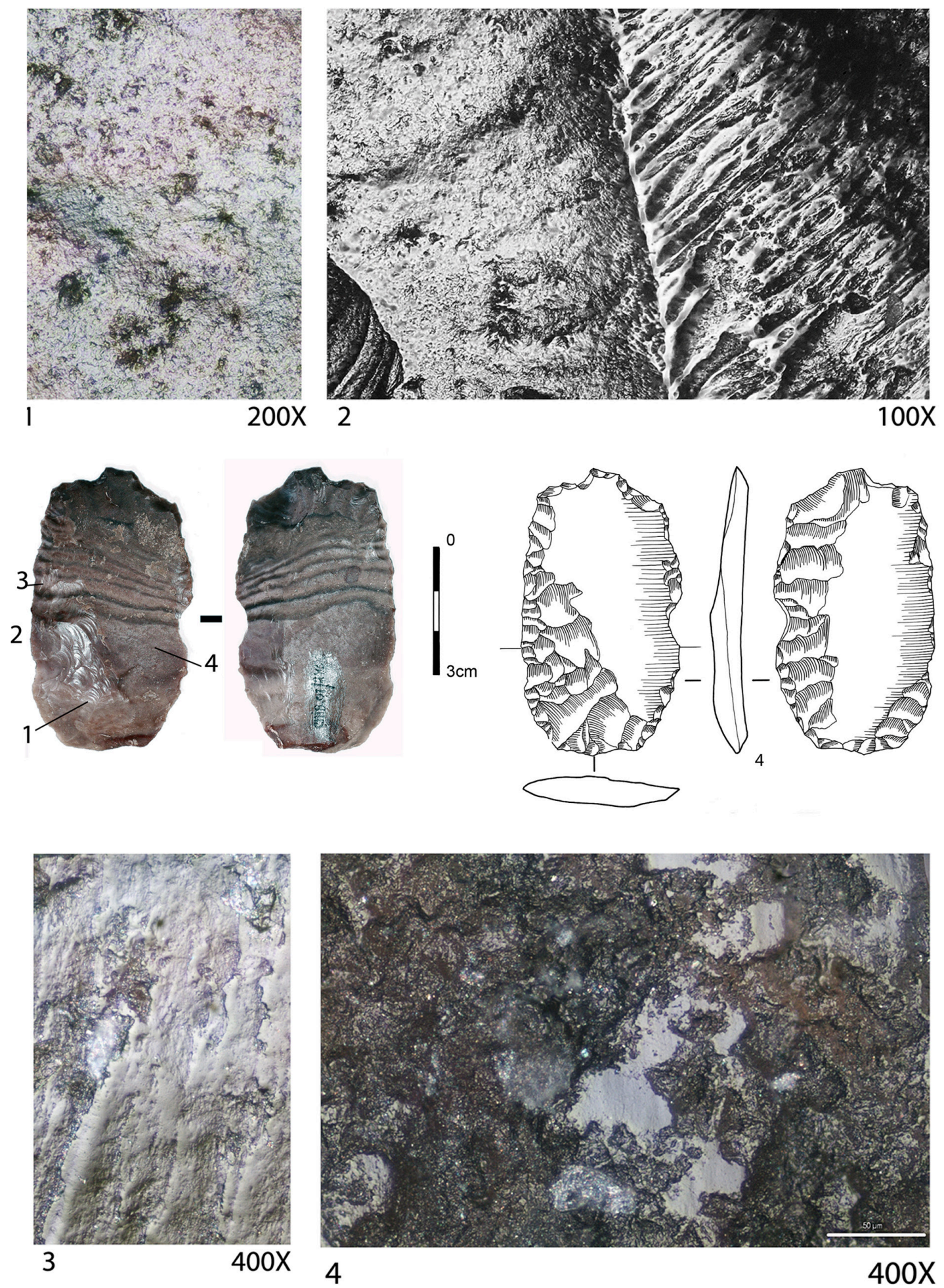

Fig. 6. Elemento de hoz en sílex (Chib. 01-2453), correspondiente al Calcolítico del poblado fortificado de Chibanes (término municipal de Palmela, Portugal): 1 superficie con lustre térmico; 2 diferencia entre superficies con micropulido (derecha) y con lustre térmico por reavivado del filo (izquierda); 3 micropulido por el uso como hoz; 4 pulido tipo ' $G$ ' o en espejo atribuido al enmangue de la pieza. Dibujo de Fernanda de Sousa.

Trab. prehist., 71, N. ${ }^{\circ}$, julio-diciembre 2014, pp. 330-342, ISSN: 0082-5638 doi: $10.3989 /$ tp.2014.12137 
Gurova 2001; Anderson et al. 2004; Gibaja-Bao et al. 2012). Estos instrumentos (Fig. 2: 7 y 8; Fig. 7) son diferentes entre sí: uno es una lasca sin retoque y el otro tiene un filo formatizado pero sin tratamiento térmico. Los filos activos son muy abruptos, no son cortantes. En las aristas fuertemente redondeadas se desarrolla un pulido rugoso con algunas zonas más lisas donde se observa mejor el contacto con la materia vegetal (Fig. 7).

Se han analizado también dos instrumentos pertenecientes al nivel campaniforme, o Grupo Palmela, del $3^{\text {er }}$ cuarto del III milenio cal. BC. Uno es un cuchillo cuyo filo derecho cortó un recurso animal blando (piel-carne). Es una lasca sin calentamiento térmico (Fig. 2: 12). El otro sí presenta el típico lustre térmico en un retoque plano y profundo (Fig. 2: 11), pudiendo indicar la continuidad de la técnica tecnológica de tratamiento térmico de las lascas. Como en el Calcolítico, cuando se fracturan esas lascas durante la elaboración, no se emplean para cortar plantas si no que pueden pasar a ser instrumentos de trabajo de otros procesos productivos y/o a no utilizarse en ninguno de ellos.
Por último, dos artefactos denticulados de los niveles del Bronce antiguo (final del III-inicios del II milenio cal. BC) muestran claros rastros de su empleo como elementos de hoz (Fig. 3). El denticulado es producto de los distintos reavivados de los filos. Su forma coincide con la de los elementos de hoz estudiados en niveles del Calcolítico/Edad del Bronce de la Meseta y sureste de la Península Ibérica (Clemente-Conte et al. 1999; Gibaja-Bao et al. 2012). Estos elementos de hoz denticulados seguirán registrándose en contextos del II milenio. Tendrán una amplia distribución geográfica, especialmente en el suroeste de la Península Ibérica (Ramos Muñoz et al. 2010: Fig. 6).

\section{DISCUSIÓN/ CONCLUSIÓN}

El análisis de los instrumentos líticos de Chibanes relacionados con prácticas de siega y procesado de cereales ha documentado un cambio interesante en los mismos entre el Calcolítico y la Edad del Bronce. En la 1. ${ }^{\mathrm{a}}$ mitad del III milenio $\mathrm{BC}$ las hoces o elementos de hoz son grandes
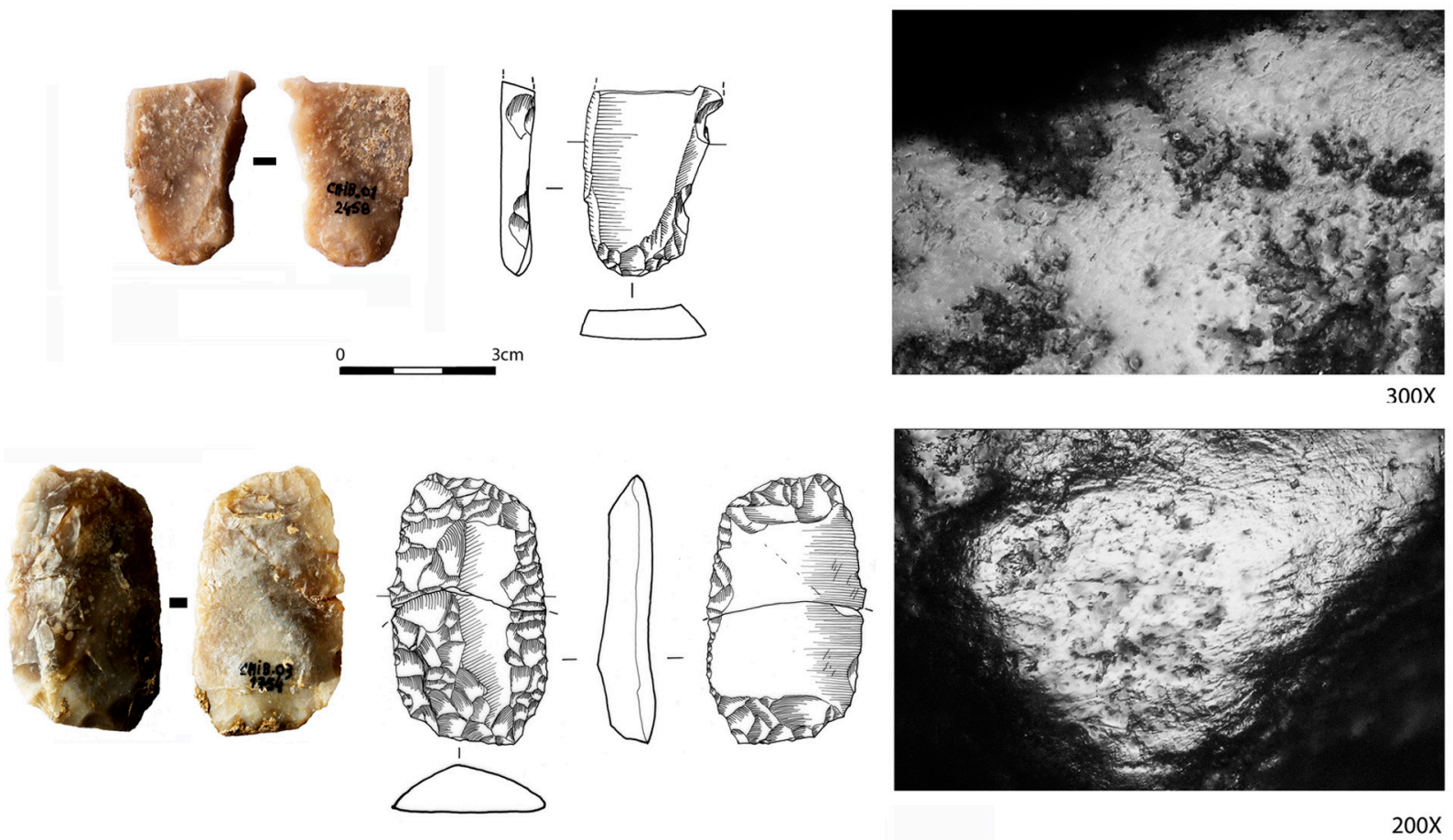

Fig. 7. Probables elementos de trillo (Chib. 01-21168 y Chib. 03-1754) en lascas de sílex, correspondiente al Calcolítico del poblado fortificado de Chibanes (término municipal de Palmela, Portugal). Dibujo de Fernanda de Sousa. 
soportes (lascas) calentados (Tratamiento Térmico) para practicarles un retoque profundo y plano. Este retoque, previo al enmangue y primer uso de los filos activos, se sigue aplicando para reavivarlos cuando se embotan por el trabajo. Su finalidad es conseguir extracciones más largas con menor fuerza de la necesaria sin el calentamiento previo. Normalmente, su aplicación para el retoque y/o la talla exige controlar la temperatura a la que se somete el soporte para evitar alteraciones (fracturas, resquebrajaduras, 'cúpulas' térmicas etc.) que inutilicen el instrumento. Todos ellas, registradas en la mayoría de estos soportes arqueológicos, son debidas al contacto con el fuego una vez abandonados o descartados. Normalmente los artefactos líticos enmangados con ayuda de almácigas o colas con resinas, como las hoces y las puntas de flecha, se reemplazan calentándolos para desprenderlos del mango. Si el reemplazo es definitivo suelen abandonarse en el fuego donde se ha realizado esa actividad.

En la 2. ${ }^{a}$ mitad del III milenio cal. BC parece que las hoces continúan haciéndose como en la fase precedente, es decir, usando el tratamiento térmico para aplicar retoques planos e invasivos en las lascas. Sin embargo, a finales del III milenio e inicios del II milenio, en la Edad del Bronce antiguo, la morfología de las hoces cambia al utilizarse lascas con un filo denticulado. El uso suele ser muy intenso, pues los filos activos aparecen muy redondeados y los pulidos muy desarrollados.

En dos de las piezas analizadas de la fase calcolítica de Chibanes, como ocurre en otros yacimientos de la Meseta ibérica, se documenta el uso del trillo (tribulum) para la separación del grano. Probablemente el trillo era un instrumento compuesto por numerosas lascas de sílex cuya conservación o almacenamiento pudo haberse hecho en otras áreas del sitio. Estos elementos de trillo se diferencian claramente de los utilizados como hoces: no se aplica el tratamiento térmico y la parte activa no resulta tan aguda y cortante sino que es mucho más roma. Sin embargo, uno de ellos presenta un trabajo secundario del filo contrario, así como de la parte proximal y distal que recuerda a los elementos de trillo etnográficos y a los arqueológicos de la zona de Bulgaria (Gurova 2001). También se han documentado trabajos muy específicos de los soportes usados a modo de trillo tanto a inicios del III milenio en Próximo Oriente (Anderson e Inizan 1994) como en el Calcolítico de la provincia de Valladolid en el interior de la Península Ibérica (Gibaja-Bao et al. 2012).

La exposición de este trabajo ha dejado patente que el análisis funcional de algunos de los materiales líticos de Chibanes ha determinado actividades relacionadas con los procesos productivos de los cereales. Su identificación como instrumentos de producción permite relacionarlos con la intensidad e importancia de la agricultura durante el Calcolítico y la Edad del Bronce. Además se ha identificado un cambio o evolución de estos instrumentos en los distintos períodos históricos analizados.

\section{AGRADECIMIENTOS}

Al personal técnico del museo de Setúbal por la acogida y constante ayuda durante la estancia en sus instalaciones para el análisis de los materiales arqueológicos. A Fernanda de Sousa por la calidad de los dibujos que presentamos en las figuras de este trabajo y a las personas encargadas de evaluar este trabajo ya que sus aportaciones y correcciones realizadas han supuesto una mejora en el resultado final.

\section{BIBLIOGRAFÍA}

Amaro, G. 2004/2005: "Interpretação das facas ovóides (foicinhas) através do estudo dos exemplares de Vila Nova de S. Pedro". Arqueologia y História 56/57: 63-80.

Anderson, P. C.; Chabot, J. y van Gijn, A. 2004: "The functional Riddle of 'Glosy' Canaanean Blades and the Near Threshing Sledge”. Journal of Mediterranean Archaeology 17-1: 87-130.

Anderson, P. C. e Inizan, M. L. 1994: "Utilisation du tribulum au début du IIIe millénaire: des lames 'cananéennes' lustrées à Kutan (Ninive V) dans la région de Mossoul, Iraq". Paléorient 20-2: 85-103.

Cardoso, J. L; Rodrigues, J. S.; Monjardino, J. y Carreira, J. R. 1986: "A jazida da Idade do Bronze Final da Tapada da Ajuda". Lisboa-Revista Municipal II, 15: 3-18.

Cardoso, J. L.; Soares, J. y Tavares da Silva, C. 1983/84: "O povoado calcolítico de Leceia (Oeiras). 1. ${ }^{\mathrm{a}}$ e 2. ${ }^{\mathrm{a}}$ campanhas de escavação (1982 e 1983)". Clio/Arqueologia 1: 41-68.

Cardoso, J. L.; Soares, J. y Tavares da Silva, C. 1996: "A ocupação neolítica de Leceia (Oeiras). Materiais

Trab. prehist., 71, N. ${ }^{\circ}$ 2, julio-diciembre 2014, pp. 330-342, ISSN: 0082-5638

doi: $10.3989 /$ tp.2014.12137 
recolhidos em 1987 e 1988". Estudos Arqueológicos de Oeiras 6: 47-89.

Carvalho, A. 1996: "O talhe de pedra e a transição Neolítico-Calcolítico no Centro e Sul de Portugal: Tecnologia e aspectos da organização da produção". Trabalhos de Arqueologia da Estudo Arqueológico da Bacia do Mondego 3/4, Colibri. Lisboa: 41-60.

Clemente-Conte, I. 1995: "Sílex y lustre térmico en el Paleolítico Medio: ¿alteración o técnica de talla? El ejemplo de Mediona I (Alt Penedès, Barcelona)". 1. ${ }^{\circ}$ Congresso de Arqueologia Peninsular, Trabalhos de Antropologia e Etnologia 35 (3): 7-43. http://hdl.handle.net/10261/47869.

Clemente-Conte, I. 1997: "Thermal alterations of flint implements and the conservation of micro-wear polish: preliminary experimental observations". En A. Ramos Millán y M. A. Bustillo (eds.): Siliceous Rocks and Culture. Universidad de Granada. Granada: 525-535.

Clemente-Conte, I. y Gibaja-Bao, J. F. 1995: "Distintos procesos de trabajo sobre recursos vegetales (cereales). Una aproximación a través de los rastros de uso". Cultures i Medi de la Prehistòria a l'Edat Mitjana. Xè Col.loqui Internacional d'Arqueologia de Puigcerdà (Puigcerdà i Osseja 10-12 de noviembre de 1994): 119-124. Patronat Francesc Eiximenis i Ajuntament de Puigcerdà. Puigcerdà.

Clemente-Conte, I. y Gibaja-Bao, J. F. 1998: "Working processes on cereals: an approach though microwear analysis". Journal of Archaeological Science 25: 457-468.

Clemente-Conte, I.; Gibaja-Bao, J. F. y Vila-Mitjà, A. 1999: "Análisis Funcional de la Industria Lítica procedente de los sondeos de Gatas". En P. V. Castro Martínez, R. Chapman, S. Gili, V. Lull, R. Micó, C. Rihuete, C. Risch y M. ${ }^{\mathrm{a}}$ E. Sanahuja (eds.): Proyecto Gatas 2. La dinámica arqueológica de la ocupación prehistórica. Arqueología Monografías 4. Junta de Andalucía, Consejería de Cultura. Sevilla: 341-347. http://hdl.handle.net/10261/48419.

Gibaja-Bao, J. F. y Clemente-Bao, I. 1996: "Análisis Funcional del material lítico en las sepulturas de la Bòbila Madurell (Sant Quirze del Vallès, Barcelona)". 1er. Congrès del Neolitic a la Peninsula Ibèrica. Formació i implantació de les comunitats agricoles. Rubricatum, revista del Museu de Gavà. 1 (1): 183-189. http://hdl.handle.net/10261/45965

Gibaja-Bao, J. F. y Clemente-Bao, I. 1997: "El tratamiento térmico del sílex y sus repercusiones en la determinación de los rastros de uso. Algunos ejemplos del neolítico en Cataluña". Revista de Arqueología de Ponent 7: 153-160. https://digital. csic.es/handle/10261/47370

Gibaja-Bao, J. F.; Clemente-Conte, I. y Vila Mitjà, A. 1997: "Una aproximación a través del Análisis Funcional a sociedades neolíticas del Noreste Peninsular". En R. de Balbín Behrmann y P. Bueno
Ramírez (eds.): Actas del II Congreso de Arqueología Peninsular (Zamora 24-27 septiembre 1996) II, Neolítico, Calcolítico y Bronce: 129-136. Zamora.

Gibaja, J. F.; Crespo, M.; Delibes de Castro, G.; Fernández, J.; Fraile, C.; Herrán, J. I.; Palomo, A. y Rodríguez, J. A. 2012: "El uso de trillos durante la Edad del Cobre en la Meseta española. Análisis traceológico de una colección de denticulados de sílex procedentes del 'recinto de fosos' de El Casetón de la Era (Villalba de los Alcores, Valladolid)". Trabajos de Prehistoria 69 (1): 133-148.

Gurova, M. 2001: "Eléments de tribulum de Bulgarieréferences ethnographiques et contexte préhistorique". Archaeologia Bulgaria V-1: 1-19.

Larsen, E. y Rainey, F. 1948: "Ipiutak and the Artic whale hunting culture". Anthropological Papers of the American Museum of Natural History 42. American Museum of Natural History. Nueva York.

Moss, E. H. 1987: "Polish G and the Question of Hafting". D. Stordeur (ed.): La main et l'outil. Manches et enmanchements préhistoriques. Travaux de la Maison de l'Orient 15. Lyon: 97-101.

Paço, A.; Bartholo, M. L. y Brandão, A. 1959: "Novos achados arqueológicos das grutas de Cascais". Actas e Memórias do I Congresso Nacional de Arqueologia (Lisboa 1958). Junta Nacional de Educação 1: 147-159. Lisboa.

Queiroz, P. F. 1999: Ecologia histórica da paisagem do noroeste alentejano. Dissertação de doutoramento apresentada à Faculdade de Ciências da Universidade de Lisboa. http://www.academia.edu/1333406/ Ecologia_historica_da_paisagem_do_Noroeste_ Alentejano (consulta 16-VII-2014).

Ramos Muñoz, J.; Pérez Rodríguez, M. y DomínguezBella, S. 2010: "Estado actual del conocimiento de las sociedades clasistas iniciales en la Banda Atlántica de Cádiz (3. ${ }^{\circ}-2{ }^{\circ}$ milenios a.n.e.)". En V. S. Gonçalves y A. C. Sousa (eds.): Transformação e mudança no centro e sul de Portugal: o $44^{\circ}$ e o 3. ${ }^{\circ}$ milenios a.n.e. Câmara Municipal de Cascais e Uniarq. Cascais: 471-487.

Rots, V. 2010: Prehension and Hafting Traces on Flint Tools. A Methodology. Leuven University Press. Leuven.

Santos, F. J. C.; Arez, L.; Monge Soares, A. M.; Deus, M. de; Queiroz P. F.; Valério, P.; Rodrigues, Z.; Antunes, A. S. y Araújo, M. de F. 2008: "Casarão da Mesquita 3 (S. Manços, Évora): um sítio de fossas 'silo' do Bronze pleno/final na encosta do Albardão". Revista Portuguesa de Arqueologia 11 (2): 55-86.

Serrao, E. da Cunha 1978: "Limitação do método comparativo na interpretação funcional dos testemunhos arqueológicos. Alguns exemplos". Actas das III Jornadas Arqueológicas (Associação dos Arqueólogos Portugueses, Lisboa, 1977) I: 15-24. Lisboa. 
Soares, J. 2003: Os hipogeus pré-históricos da Quinta do Anjo (Palmela) e as economias do simbólico. Museu de Arqueologia e Etnografia do Distrito de Setúbal. Setúbal (MAEDS).

Soares, J. 2014: Transformações sociais durante o III milénio AC no Sul de Portugal. O povoado do Porto das Carretas. EDIA, DRCAL e MAEDS. Lisboa.

Soares, J. y Tavares da Silva, C. 1975: “A ocupação pré-histórica do Pedrão e o Calcolítico da região de Setúbal”. Setúbal Arqueológica 1: 53-154.

Soares, J. y Tavares da Silva, C. 1984: "Le Groupe de Palmela dans le cadre de la céramique campaniforme au Portugal". En J. Guilaine (ed.): L'Áge du Cuivre Européen. Civilisations à Vases Campaniformes. Centre National de la Recherche Scientifique. Toulouse: 209-220.

Soares, J. y Tavares da Silva, C. 2010: "Campaniforme do Porto das Carretas (Médio Guadiana). A procura de novos quadros de referencia". En V. S. Gonçalves y A. C. Sousa (eds.): Transformação e mudança no Centro e Sul de Portugal: o $4 .^{\circ}$ e o 3. ${ }^{\circ}$ milénios a.n.e. Câmara Municipal de Cascais e Uniarq. Cascais: 225-261.
Tavares da Silva, C. y Soares, J. 1978: "Uma jazida do Bronze final na Cerradinha (Lagoa de Santo André, Santiago do Cacém)". Setúbal Arqueológica 4: 71-115.

Tavares da Silva, C. y Soares, J. 1997: "Chibanes revisitado. Primeiros resultados da campanha de escavações de 1996". Estudos Orientais 6: 33-66.

Tavares da Silva, C. y Soares J. 2012: "Castro de Chibanes (Palmela). Do III milénio ao séc. I a.C.”. En I. C. F. Fernandes e M. Teixeira Santos (eds.): Palmela arqueológica no contexto da região interestuarina Sado-Tejo. Câmara Municipal de Palmela. Palmela: 67-87.

Tavares da Silva, C. y Soares J. 2014: "O Castro de Chibanes (Palmela) e o tempo social do III milenio $\mathrm{BC}$ na Estremadura". II Encontro de Arqueologia da Arrábida. Homenagem a A. I. Marques da Costa, Setúbal Arqueológica 15: 105-172.

Tereso, J. P. 2014: "Vestígios arqueobotânicos do III milénio cal BC de Chibanes (Palmela, Setúbal)”. II Encontro de Arqueologia da Arrábida. Homenagem a A. I. Marques da Costa, Setúbal Arqueológica 15: 173-180. 